\title{
HMG Domain
}

National Cancer Institute

\section{Source}

National Cancer Institute. HMG Domain. NCI Thesaurus. Code C13422.

Each HMG subfamily is a distinct set of proteins with identifiable structural characteristics and a specific type of targets to induce characteristic changes in the structure of its binding site. 